\title{
Development of Biased Diamond Current Amplifier
}

\author{
Joan E. Yater, Jonathan L. Shaw, \\ Kevin L. Jensen, and Frank Wood \\ Electronics Science and Technology Division \\ Naval Research Laboratory \\ Washington, DC 20375 \\ joan.yater@nrl.navy.mil \\ Jeremy Hanna \\ Beam-Wave Research, Inc. \\ Bethesda, MD 20814
}

\author{
Tatyana Feygelson \\ Science Applications International Corp. \\ Washington, DC 20003
}

\author{
Bradford B. Pate \\ Chemistry Division \\ Naval Research Laboratory \\ Washington, DC 20375
}

\begin{abstract}
A diamond current amplifier is being developed that can be biased to generate an internal electric field needed for high transport efficiency and emission gain. In the process, a bonded diamond structure has been successfully fabricated that provides mechanical support for the microns-thick diamond film and that allows for the use of processing techniques needed to fabricate the biased device. Emission measurements taken from the hydrogenated surface of a 5.4-micron-thick bonded diamond film reveal a temperature-dependent behavior that suggests the presence of upwards band bending at the surface. Studies are in progress to identify the cause of the band bending at the hydrogenated surface and to develop a better understanding of the surface electronic and material properties that influence the amplifier performance.
\end{abstract}

Keywords: secondary emission; diamond; current amplifier; negative electron affinity; electron transport.

\section{Introduction}

High-current-density cathodes are required for the development of high-power, high-frequency vacuum electronic devices, as well as for other electron beam applications. To address this need, a current amplifier stage is being developed that will multiply an incident electron current (via the secondary-electron multiplication process) and then emit the amplified beam so as to achieve a current gain of 50-100. Diamond is a particularly promising current amplification source due to the negative electron affinity (NEA) present at the hydrogenated surface. As such, we are fabricating current amplifiers using singlecrystal CVD diamond films grown at NRL. In order to obtain the highest possible gain, very good electron transport and emission efficiency must be achieved in the diamond films. Therefore, our growth effort is focused on reducing impurity and defect concentrations in thin freestanding CVD films and on preparing emission surfaces with uniform NEA and low roughness.

In our initial studies [1], an 8.3-micron-thick freestanding single-crystal CVD diamond film was mounted in a machined sample holder, and secondary-electron-emission (SEE) measurements were used to examine the transport and emission characteristics of the unbiased film. Lowenergy electron transmission was successfully demonstrated from the unbiased diamond film, with the electron energy distribution dominated by a sharp, narrow $(\mathrm{FWHM}=0.35 \mathrm{eV})$ emission peak. In fact, low-energy secondary electrons were emitted from the conduction band even after diffusing through the entire 8.3-micron-thick film. Although this demonstrated the potential for efficient transport, the maximum transmission gain of $\sim 3-4$ was limited by diffusive transport in the field-free measurements. Therefore, the current amplifier must employ a biased operation to achieve the higher transport efficiency needed for high-gain amplification.

\section{Biased Current Amplifier Approach}

In our first approach, an 8.5-micron-thick diamond film was mounted in a sample holder that could be biased. While the transmission characteristics under unbiased conditions were similar to those observed with the previous 8.3-micron-thick sample, problems with leakage current and poor electrical contact were encountered upon biasing the amplifier. Unfortunately, such problems could not be avoided in spite of our efforts. As such, our approach is now focused on bonding the diamond film to a larger diamond substrate so that amplifier structures can be fabricated that provide more controlled biasing capabilities.

Figure 1 shows a schematic of the bonded diamond amplifier that is being fabricated for biased operation. The first step is to bond the diamond film to a large-area polycrystalline diamond substrate that has been prepared by

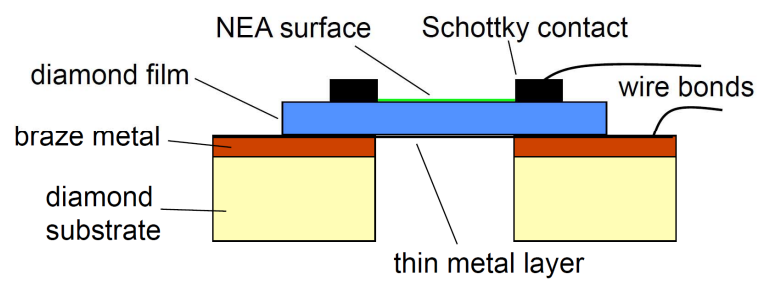

Figure 1. Schematic of bonded diamond amplifier with metal contacts that provide biasing capabilities. 
laser cutting a center hole for the incident beam. Prior to bonding, a thin metal layer is sputter deposited onto the backside of the diamond film, and braze metal is sputter deposited onto the substrate. The assembled diamond is then bonded in a heated vacuum oven. The next step is to deposit a patterned Schottky metal contact on the front side of the diamond film to allow for the necessary biasing, and to hydrogenate the exposed surface to create an NEA emitting region for the transmitted electrons. Efforts are presently underway to develop a lithographic technique to deposit the contact and to optimize the patterned structure as needed to achieve uniform near-surface fields.

\section{Emission Characterization}

The first bonded amplifier to be studied was a 5.4-micronthick, high-purity diamond film. This film was hydrogenated but not metallized on the front side, and therefore the diamond amplifier could not yet be biased. As such, emission measurements were taken under unbiased conditions, with the front hydrogenated surface electrically floating. The measurements were taken in both transmission and reflection configurations. In transmission mode, high-energy electrons are injected into the diamond film using a $0-20 \mathrm{keV}$ electron gun, and the transmitted low-energy electrons are analyzed to provide transport information. In reflection mode, SEE measurements are taken from the front surface using a lower-energy gun $(0-1 \mathrm{keV})$ to examine the surface emission characteristics. The data can also be analyzed using Monte Carlo simulations [2] to gain insight into the transport / emission properties.

In our measurements from the bonded 5.4-micron-thick diamond film, the reflection yields were very low but relatively constant $(\sim 1)$ at all beam energies $E_{0}=0-1 \mathrm{keV}$, while no transmission current was detected. Furthermore, the energy distribution curves (EDCs) taken in reflection mode were shifted higher in energy compared to our previous study. These observations suggested the presence of upwards band bending and surface charging that would prevent the emission of very-low-energy electrons.

Moreover, the measurements revealed a temperaturedependent behavior not observed previously. The EDCs shown in Fig. 2 were taken while the diamond was heated from $30^{\circ} \mathrm{C}$ to $200^{\circ} \mathrm{C}$, and the heating clearly produces a strong downward shift in the emission onset energy along with the emergence of a sharp emission peak. Such behavior is consistent with downwards band bending induced by the thermal activation of B impurities (however few). Before heating, the EDCs also displayed a significant upward shift at lower values of $\mathrm{E}_{\mathrm{o}}$ (Fig. 3, upper plot), which is consistent with beam-induced charging. However, as the temperature increased (along with electrical conductivity), this energy shift was eliminated (Fig. 3, lower plot) and slightly higher yields were produced (note the expanded scale in upper plot).

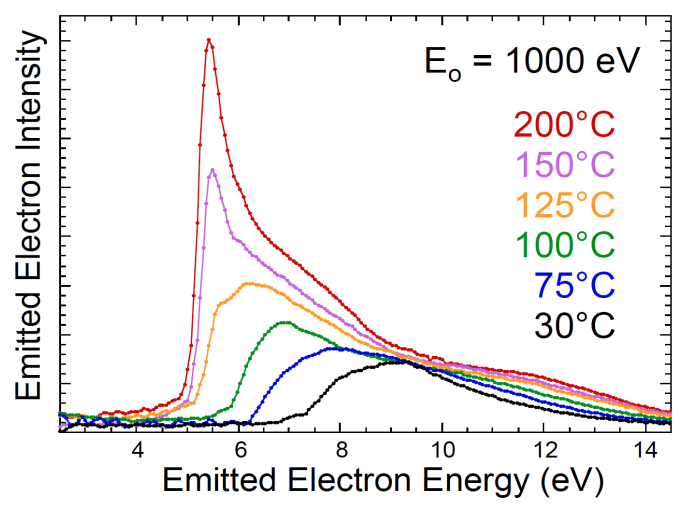

Figure 2. Reflection EDCs measured from the hydrogenated diamond surface during heatings to successively higher temperatures.

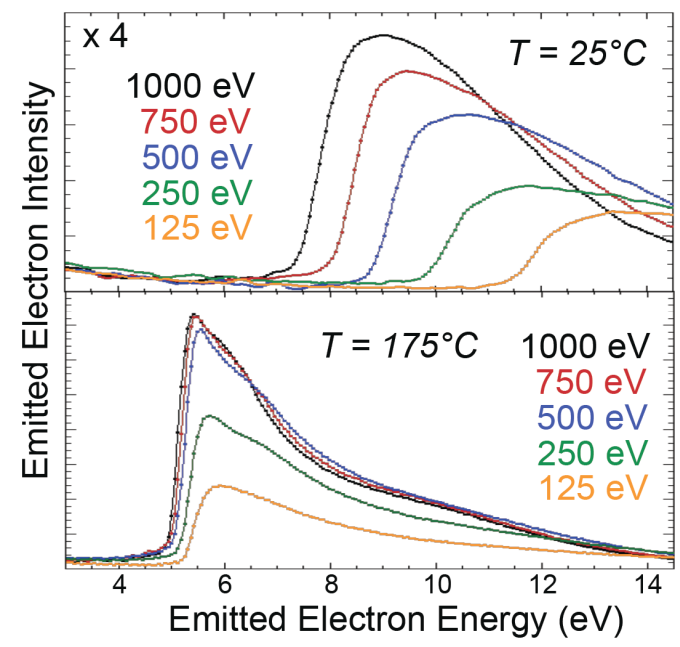

Figure 3. Reflection EDCs taken as a function of $E_{0}$ before heating (top) and during heating (bottom).

Fortunately, the observed band bending should be removed by the presence of the front-side contact and the internal electric field. Until then, studies are focused on identifying the cause of the band bending since this will provide a better understanding of the surface electronic and material properties that influence the amplifier performance.

\section{Acknowledgements:}

This work is supported by the Office of Naval Research.

\section{References}

1. Yater, J.E., J.L. Shaw, K.L. Jensen, T. Feygelson, R.E. Myers, B.B. Pate, and J.E. Butler, "Secondary Electron Amplification Using Single-crystal CVD Diamond Film," Diamond and Related Materials, vol. 20, pp. 798-802, May/June 2011.

2. Jensen, K.L., J.E. Yater, J.L. Shaw, R.E. Myers, B.B. Pate, J.E. Butler, and T. Feygelson, "Bunch Characteristics of an Electron Beam Generated by a Diamond Secondary Emitter Amplifier," Journal of Applied Physics, vol. 108, pp. 044509, Aug. 2010. 